\title{
Potassium Channel Kir4.1 Macromolecular Complex in Retinal Glial Cells
}

\author{
NATHAN C. CONNORS ${ }^{1}$ AND PAULO KOFUJI ${ }^{2 *}$ \\ ${ }^{1}$ Department of Neuroscience, University of Pennsylvania, Philadelphia, Pennsylvania \\ ${ }^{2}$ Department of Neuroscience, University of Minnesota, Minneapolis, Minnesota
}

\section{KEY WORDS}

potassium buffering; dystrophin; syntrophin; Kir4.1; AQP4; Müller cells; astrocytes

\begin{abstract}
A major role for Müller cells in the retina is to buffer changes in the extracellular $\mathrm{K}^{+}$concentration $\left(\left[\mathrm{K}^{+}\right]_{\mathrm{o}}\right)$ resulting from light-evoked neuronal activity. The primary $\mathrm{K}^{+}$conductance in Müller cells is the inwardly rectifying $\mathrm{K}^{+}$channel Kir4.1. Since this channel is constitutively active, $\mathrm{K}^{+}$can enter or exit Müller cells depending on the state of the $\left[\mathrm{K}^{+}\right]_{0}$. This process of $\left[\mathrm{K}^{+}\right]_{\mathrm{o}}$ buffering by Müller cells (" $\mathrm{K}^{+}$siphoning") is enhanced by the precise accumulation of these $\mathrm{K}^{+}$channels at discrete subdomains of Müller cell membranes. Specifically, Kir4.1 is localized to the perivascular processes of Müller cells in animals with vascular retinas and to the endfeet of Müller cells in all species examined. The water channel aquaporin-4 (AQP4) also appears to be important for $\left[\mathrm{K}^{+}\right]_{\mathrm{o}}$ buffering and is expressed in Müller cells in a very similar subcellular distribution pattern to that of Kir4.1. To gain a better understanding of how Müller cells selectively target $\mathrm{K}^{+}$and water channels to discrete membrane subdomains, we addressed the question of whether Kir4.1 and AQP4 associate with the dystrophin-glycoprotein complex (DGC) in the mammalian retina. Immunoprecipitation (IP) experiments were utilized to show that Kir4.1 and AQP4 are associated with DGC proteins in rat retina. Furthermore, AQP4 was also shown to co-precipitate with Kir4.1, suggesting that both channels are tethered together by the DGC in Müller cells. This work further defines a subcellular localization mechanism in Müller cells that facilitates $\left[\mathrm{K}^{+}\right]_{\mathrm{o}}$ buffering in the retina. $\odot 2005$ Wiley-Liss, Inc.
\end{abstract}

\section{INTRODUCTION}

The inwardly rectifying potassium channel Kir4.1 is a tetrameric transmembrane protein expressed in multiple organ systems. Within the central nervous system (CNS) Kir4.1 is expressed in glia, including the principal glial cell type in the retina, the Müller cell (Kofuji et al., 2000). Kir4.1 is implicated in the process of "potassium siphoning" (Newman and Odette, 1984), a mechanism of potassium buffering that helps stabilize the extracellular potassium concentration $\left(\left[\mathrm{K}^{+}\right]_{0}\right)$ in the face of neuronallyderived ion fluxes (Amedee et al., 1997; Kofuji et al., 2000; Kofuji and Connors, 2003).

Müller cells have a bipolar morphology and are arranged in a radial fashion, spanning almost the entire width of the neural retina. A unique property of Kir4.1 within these cells is its subcellular localization, being highly concentrated in Müller cell endfeet and in perivascular processes (Nagelhus et al., 1999; Kofuji et al., 2000). This localization pattern is thought to facilitate the potassium siphoning mechanism by enrichment of Kir4.1 in cellular regions where $\mathrm{K}^{+}$can be released from Müller cells without altering the stability of neuronal physiology (Odette and Newman, 1988; Newman and Reichenbach, 1996).

Interestingly, the water channel aquaporin-4 (AQP4) maintains a very similar distribution pattern to that of Kir4.1 in Müller cells (Nagelhus et al., 1999). Recent findings suggest that AQP4 may also be necessary for effective $\left[\mathrm{K}^{+}\right]_{\mathrm{o}}$ buffering in brain (Amiry-Moghaddam et al., 2003a; Nagelhus et al., 2004). The co-localization of this channel with Kir4.1 in Müller cells suggests a common molecular mechanism for their consolidation in these subcellular regions (Nagelhus et al., 2004).

Studies by our group and others have revealed that both Kir4.1 (Connors and Kofuji, 2002; Connors et al., 2004) and AQP4 (Neely et al., 2001; Amiry-Moghaddam et al., 2003a) can interact with the multi-protein dystrophin-glycoprotein complex (DGC) in brain and astrocytes. This group of proteins has been best described in muscle, where it may be involved in calcium homeostasis or the maintenance of structural integrity required for myocytes to withstand muscular contractile forces (Mehler, 2000). A specific arrangement of the DGC in Müller cells was recently described (Claudepierre et al., 2000). At its core, this complex includes the PDZ domain protein $\alpha$-syntrophin, the short dystrophin isoform Dp71, the transmembrane protein $\beta$-dystroglycan, and the extracellular matrix receptor $\alpha$-dystroglycan (Claudepierre et al., 2000).

Recent studies have shown that mutations in DGC proteins underlie a variety of muscular dystrophies (Michele and Campbell, 2003). Such mutations also result in structural and physiological changes within the CNS, revealing an important role for the DGC in brain function. For example, a mutant form of $\alpha$-dystroglycan results in structural anomalies within the brain and prevents the onset of hippocampal LTP (Moore et al., 2002). Dystro-

Grant sponsor: National Institutes of Health; Grant numbers: EY12949,
EY07133.
*Correspondence to: Paulo Kofuji, Department of Neuroscience, University of
Minnesota, 6-145 Jackson Hall, 321 Church St. SE, Minneapolis, MN 55455 .
E-mail: kofuj001@tc.umn.edukofuj001@tc.umn.edu
Received 9 February 2005; Accepted 19 July 2005
DOI 10.1002/glia.20271
Published online 3 October 2005 in Wiley InterScience (www.interscience. Published
wiley.com) 
phin mutants also show abnormal electroretinogram (ERG) profiles (Pillers et al., 1999a,b; Dalloz et al., 2003), and the elimination of Dp71 results in the mislocalization of both Kir4.1 and AQP4 in mouse retina (Connors and Kofuji, 2002; Dalloz et al., 2003).

Although we previously showed that Kir4.1 can be localized in Müller cells by Dp71 (Connors and Kofuji, 2002) and that Kir4.1 interacts directly with the DGC via $\alpha$-syntrophin in brain and astrocytes (Connors et al., 2004), we have yet to describe in detail the molecular interactions between the DGC and Kir4.1 in Müller cells. In this study we show that in the retina Kir4.1 can form a stable complex with members of the DGC including dystrophin, syntrophin, and dystrobrevin, and moreover can interact with AQP4 possibly by co-associations with DGC proteins.

\section{MATERIAL AND METHODS Antibodies}

Rabbit anti-Kir4.1 generated against the peptide CEKEGSALSVRISNV and characterized previously (Kofuji et al., 2000) was used as a pulldown antibody in immunoprecipitation (IP) assays ( $5 \mu \mathrm{g} /$ reaction) and as a probe for Western blots (1-2.5 $\mathrm{gg} / \mathrm{ml})$. Anti-aquaporin-4 was obtained from either Chemicon (Temecula, CA) or Alamone Laboratories (Jerusalem, Israel) and used at 0.1-0.2 $\mu \mathrm{g} / \mathrm{ml}$ for Western blotting, and $3 \mu \mathrm{g}$ for each IP pulldown. Mouse monoclonal anti-glutamine synthetase clone GS-6 (Chemicon) was used at $5 \mu \mathrm{g} / \mathrm{ml}$ for retinal slice immunocytochemistry. Guinea pig anti-GLAST (Chemicon) used for ICC at 1:4,000 of the manufacturer's stock as a Müller cell marker and as a probe for Western blots at 1:5,000. Rabbit polyclonal anti- $\alpha$-syntrophin antibody was obtained from Sigma (St. Louis, MO) and used as a pulldown antibody for IPs at 3-5 $\mu$ g per pulldown or at $7.7 \mu \mathrm{g} / \mathrm{ml}$ as a probe for Western blots. Monoclonal pansyntrophin antibodies from Affinity Bioreagents (ABR, Golden, CO) were used for Western blotting at $0.2 \mu \mathrm{g} / \mathrm{ml}$. Monoclonal anti-dystrophin (clone 6C5) from Chemicon was used at 1:20 of manufacturer stock for probing Western blots. Mouse anti- $\beta$-dystroglycan monoclonal antibody (clone 43DAG1/8D5) from Novacastra (Newcastle upon Tyne, UK) was used for immunoprecipitation assays (100 $\mu \mathrm{l} /$ reaction) and at 1:50 for probing Western blots. AlexaFluor secondary antibodies (Molecular Probes, Eugene, OR) produced in goat were used, including antirabbit 488 and 594, anti-mouse 488 and 594, and anti-guinea pig 488 and 594. For detection on Western blotting, peroxidase-conjugated anti-mouse (working concentration 1:30,000) or anti-rabbit (1:20,000) were used (Jackson ImmunoResearch, West Grove, PA).

\section{Immunocytochemistry}

Immunocytochemistry was performed with adult postnatal day 21 (P21) to P26 wild-type (C57BL/6J) mice (Jackson Laboratory, Bar Harbor, ME) using previously described procedures (Connors and Kofuji, 2002).

\section{Chemical Crosslinking, Immunoprecipitation, and Western Blotting}

Frozen rat retinas (Pel Freez, Rogers, AK) were dissected in room temperature phosphate-buffered saline (PBS) and then dropped into pre-DSP crosslink buffer $\left(250 \mu \mathrm{M}\right.$ sucrose, $7.5 \mathrm{mM} \mathrm{Na} \mathrm{HPO}_{4}, 5 \mathrm{mM}$ EGTA, $5 \mathrm{mM}$ EDTA (pH 7.4), and Complete protease inhibitor mixture (Roche Diagnostics, Indianapolis, IN)) on ice at a ratio of $1.8 \mathrm{ml}$ buffer per 30 retinas. Retinas were then homogenized on ice by repeated aspiration through a syringe and 18 gauge needle, then $2 \mathrm{mM}$ dithiobis(succinimidyl propionate) (DSP; Pierce, Rockford, IL) was dissolved in DMSO and added to the homogenate for a final concentration of $200 \mu \mathrm{M}$. Retinas were crosslinked at room temperature for $30 \mathrm{~min}$ until the reaction was stopped by the addition of Tris ( $\mathrm{pH} 7.4$ ) to a final concentration of $50 \mathrm{mM}$. The tube was then put back on ice and retinal tissue was lysed by the addition of $150 \mathrm{mM} \mathrm{NaCl}$ and $1 \%$ Triton X100 at $4^{\circ} \mathrm{C}$ for $1 \mathrm{~h}$, then spun down in a microcentrifuge at $4^{\circ} \mathrm{C}$ at $14,000 \mathrm{rpm}$ for $10 \mathrm{~min}$. The supernatant was collected and spun again, then used immediately thereafter. All the following steps were performed on ice or at $4^{\circ} \mathrm{C}$, except for centrifugations of the Sepharose beads. After retinal lysates were prepared, they were precleared by incubation for $1-3 \mathrm{~h}$ with $50 \mu \mathrm{l}$ protein A- or G-Sepharose beads, which were then removed by centrifugation and recovery of the supernatant. Lysates from 30 rat retinas were divided equally into two parts; one was spiked with the pulldown antibody, while the other was not, and both were incubated overnight on a mechanical rotator. The next day, 20-30 $\mu$ l Sepharose beads previously blocked in $2 \%$ bovine serum albumin (BSA) were added to each of the IP and the non-IP reactions and incubated on the rotator for $1 \mathrm{~h}$. Following incubation, a small amount of the non-IP (beads-only) sample was recovered for the input lane. Samples were washed in lysis buffer consisting of $50 \mathrm{mM}$ Tris ( $\mathrm{pH} 7.4$ ), $150 \mathrm{mM}$ NaCl, $50 \mathrm{mM}$ EGTA, and $0.1 \%$ Triton X-100 fortified with Complete protease inhibitor a total of 5 times. After the final wash, the Sepharose beads as well as the input sample were resuspended in NuPage loading buffer (Invitrogen) prepared according to the manufacturer's instructions, and heated at $70^{\circ} \mathrm{C}$ for $10 \mathrm{~min}$. Western blots were performed using NuPAGE BisTris 4-12\% gradient gels (Invitrogen, San Diego, CA) as described previously (Connors et al., 2004).

\section{RESULTS Kir4.1 Interacts With DGC in Retina}

Our prior research (Connors and Kofuji, 2002) demonstrated that Dp71 is critical for the subcellular localization of Kir4.1 in Müller cells within the retina. Our goal in the present study is to describe the molecular interactions involved in this localization of Kir4.1 as well as AQP4. We first performed immunostaining experiments on mouse retina to reveal the distribution of both channels. Figure 1 shows that the localization pattern of both Kir4.1 and AQP4 are very similar within the retina. Specifically, 

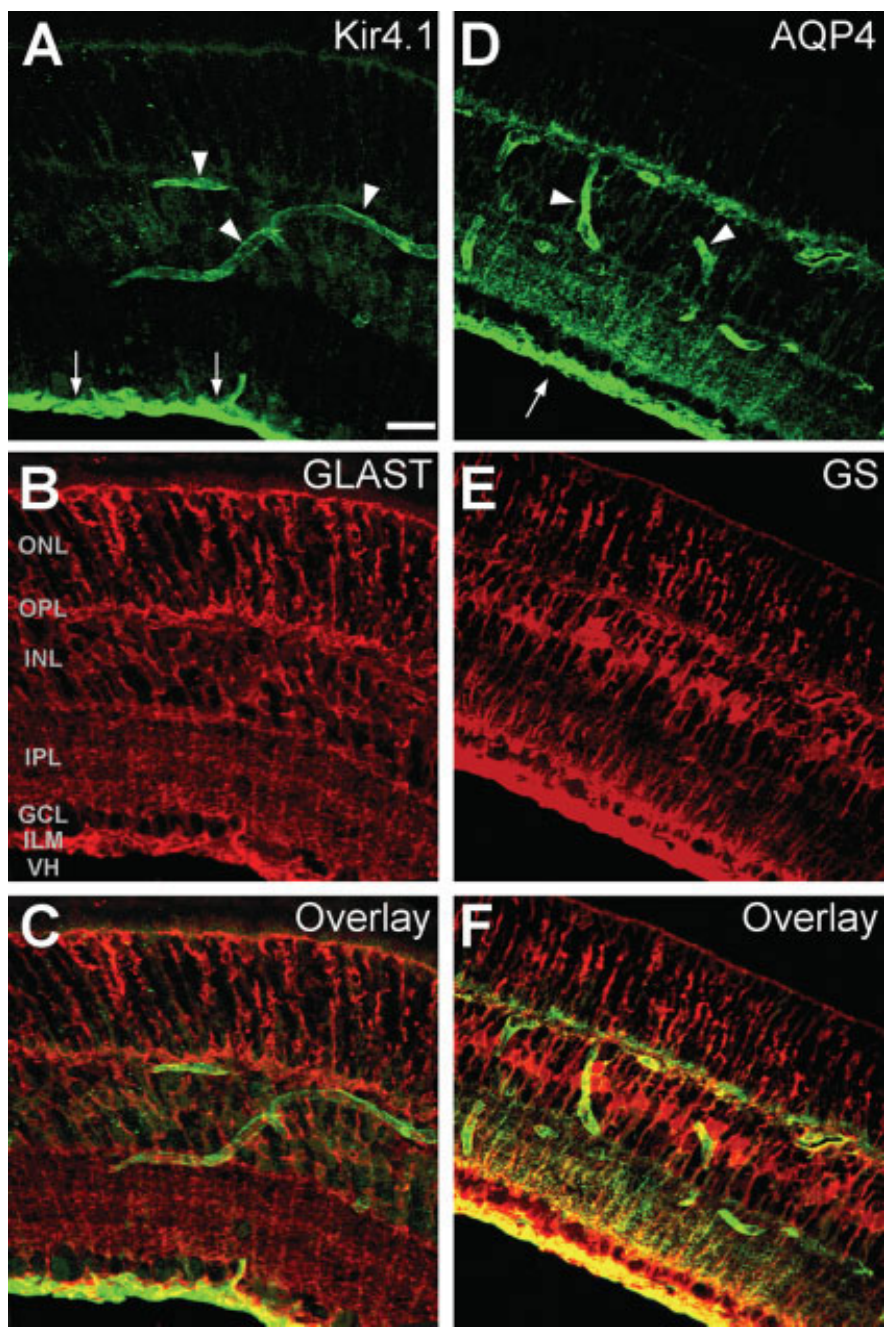

Fig. 1. Kir4.1 and AQP4 are distributed in a similar fashion in Müller glial cells. A: Mouse retinal section was probed with antibodies against Kir4.1. Note the enrichment of staining at the inner limiting membrane (ILM) where Müller cell endfeet are located (arrows) as well as in perivascular processes in the deeper retinal layers (arrowheads). B: Section shown in A was also co-stained with antibodies against the glutamate/aspartate transporter GLAST to delineate Müller cell membranes. C: Merged image of A and B. D: AQP4 immunostaining is very similar to that of Kir4.1, with an enrichment at the ILM (arrow), as well as in perivascular processes (arrowheads). E: Same section shown in D co-stained with the Müller cell marker glutamine synthetase (GS). F: Merged image of D and E. ONL, outer nuclear layer; OPL, outer plexiform layer; INL, inner nuclear layer; IPL, inner plexiform layer; GCL, ganglion cell layer; ILM, inner limiting membrane; VH, vitreous humor. Scale bar $=50 \mu \mathrm{m}$.

both channels are localized to the vitreal border, corresponding to staining within Müller cell endfeet (Fig. 1A,D, arrows) as well as in perivascular processes in outer retinal layers (Fig. 1A,D, arrowheads). Kir4.1 appears to be highly enriched in these areas as very little staining for the channel is seen elsewhere throughout the retina. The staining for AQP4 also shows this highly polarized distribution; however, notable staining can also be seen in the outer plexiform layer (OPL) and throughout the inner plexiform layer (IPL). These results are in agreement with previous findings (Dalloz et al., 2003; Nagelhus et al., 1999), confirming that Kir4.1 and AQP4 are specifically localized to particular subcellular regions within Müller cells. Similar results were also observed in rat retinas (results not shown).

Our prior data revealed that when dystrophins are absent from the retina, Kir4.1 no longer maintains such a polarized localization pattern within Müller cells, and a more homogeneous distribution is observed (Connors and Kofuji, 2002). Based on this information, we sought to determine whether the channel can directly interact with members of the DGC in the retina. We first asked whether the IP of Kir4.1 can result in the recovery of the short dystrophin isoform Dp71, implicated as the primary subtype of dystrophin in Müller cells (Claudepierre et al., 1999). We employed the crosslinking reagent DSP for the current studies to minimize instability of interactions between channel and the DGC. This reagent has previously been used by other groups to stabilize interactions between the transmembrane channels and their interaction partners (Zhu et al., 1999; Neely et al., 2001). Whole retinas from rats were isolated, chemically crosslinked, then incubated with antibodies against Kir4.1. The protein complexes recovered with protein A-Sepharose beads were eluted, processed through sodium dodecyl sulfatepolyacrylamide gel electrophoresis (SDS-PAGE) Western blotting, and then probed with antibodies against dystrophin.

The results of the above experiment showed a prominent band slightly lower than $75 \mathrm{kDa}$ when lysates were precipitated with anti-Kir4.1 (Fig. 2A, IP:Kir4.1) and probed with anti-dystrophin antibodies, indicating the recovery of Dp71. Two higher-molecular-weight bands were also seen: one slightly larger than $100 \mathrm{kDa}$ and one at $\sim 150 \mathrm{kDa}$. While Dp71 is purportedly the only dystrophin expressed in Müller cells, the larger isoforms Dp140 and Dp260 are also likely expressed in other areas of the retina (Howard et al., 1998; Claudepierre et al., 1999). The larger dystrophin-positive bands seen in Figure 2A resemble the two distinct isoforms of Dp140 (Austin et al., 1995), suggesting that Dp140 may also interact with Kir4.1 in the retina. All bands recovered in the IP lane resemble those seen in nonprecipitated lysates (Fig. 2A, input lane). Nonspecific bands were detected when pulldown antibody was omitted (Fig. 2A, pA lane), possibly arising from retinal immunoglobulins, yet the mobilities of these bands did not interfere with the detection of dystrophin-positive bands (Fig. 2A, IP:Kir4.1 lane). Together this evidence strongly suggests that dystrophins and Kir4.1 form a macromolecular complex in retina.

To help further verify whether the bands seen following the IP of Kir4.1 correspond to dystrophins, retinal lysate was also immunoprecipitated with antibodies against the known dystrophin binding partner, $\alpha$-syntrophin. Upon probing these blots with antibodies against dystrophin, bands that appeared to correspond to Dp71 and Dp140 were revealed (Fig. 2A, IP: $\alpha$-syn lane), and matched closely the bands observed in the IP of Kir4.1. This provides further evidence that the bands recovered by the IP of Kir4.1 correspond to isoforms of dystrophin.

Other IP experiments were performed to further characterize the interaction between Kir4.1 and the DGC in 


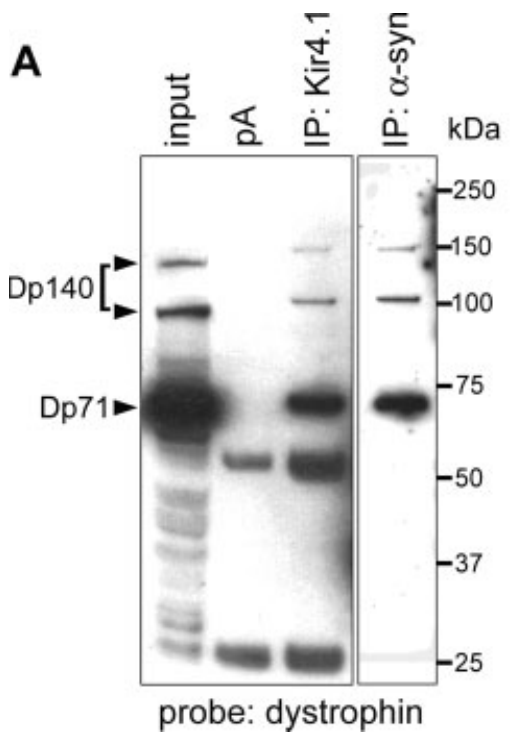

Fig. 2. Kir4.1 associates with dystrophin isoforms in rat retinas. A, When retinal tissue was immunoprecipitated with antibodies against Kir4.1 and probed with anti-dystrophin antibodies (IP:Kir4.1 lane), a number of immunopositive bands were detected. The most intense band was seen at just under $75 \mathrm{kDa}$, indicating that this protein was Dp71. Two larger bands were also seen at 100-150 kDa, likely corresponding to isoforms of Dp140. IP with antibodies against $\alpha$-syntrophin (IP: $\alpha$-syn) resulted in recovery of the same isoforms of dystrophin as did Kir4.1. B Retinal lysates were chemically crosslinked, incubated with antibodies against Kir4.1, and then processed via SDS-PAGE and Western blotting using anti-pan-syntrophin antibodies (top) and anti-pan-dystrobrevin (bottom) antibodies. Since syntrophins have a molecular weight of 59 $\mathrm{kDa}$ and dystrobrevin at $\sim 87 \mathrm{kDa}$, the bands detected in the IP lane in each blot (IP:Kir4.1) indicate the presence of these two proteins. In neither case did the protein A-Sepharose matrix nonspecifically pull down the proteins of interest. Numbers on the right refer to the relative electrophoretic mobility of pre-stained molecular mass standards.

the retina. During one such experiment, the IP of Kir4.1 resulted in the recovery of a band with a molecular weight slightly less than $75 \mathrm{kDa}$ when probed with a pan-syntrophin antibody (Fig. 2B, top). Syntrophins have been shown to migrate at $58 \mathrm{kDa}$ on SDS-PAGE (Froehner et al., 1987; Adams et al., 1995; Suzuki et al., 1995), which is consistent with the band seen on our blot.

Another DGC component, dystrobrevin, is an intracellular protein of $87 \mathrm{kDa}$ that associates with both dystrophin and syntrophin (Peters et al., 1997; Sadoulet-Puccio et al., 1997). When samples precipitated with anti-Kir4.1 were probed with antibodies against dystrobrevin, a band slightly greater than $75 \mathrm{kDa}$ was detected (Fig. 2B, bottom), suggesting that this protein also interacts with Kir4.1. In both experiments, omission of the pulldown antibody resulted in the failure to recover the DGC protein (Fig. 2B, pA lanes).

To help reinforce some of the findings outlined thus far, some reverse experiments were performed by immunoprecipitating selected DGC components, then probing for Kir4.1. We first used antibodies against Kir4.1 to pulldown the channel protein, then probed the membrane for Kir4.1 to verify the migration rate of Kir4.1 on SDSPAGE in these experiments. It has been shown consistently on Western blots that it is difficult to completely dissociate Kir4.1 from a tetrameric state (Li et al., 2001;

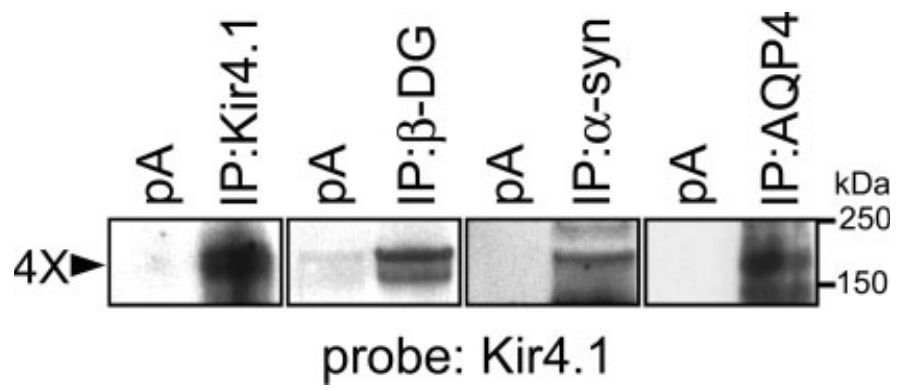

Fig. 3. Kir4.1 co-precipitates with DGC proteins and AQP4 in retina. First panel, retinal tissue was homogenized chemically crosslinked and immunoprecipitated with antibodies against Kir4.1. The sample was processed through SDS-PAGE and Western blotting and probed with antibodies against Kir4.1, showing a band at $\sim 180 \mathrm{kDa}$ corresponding to the tetrameric form of the channel. Precipitation with protein A-Sepharose only (pA, left lane) did not pull down Kir4.1. Second and third panels, when lysates were immunoprecipitated with antibodies against $\beta$-dystroglycan and $\alpha$-syntrophin, respectively, a 180 $\mathrm{kDa}$ band was seen after probing with anti-Kir4.1 antibodies. When anti-AQP4 were used as pulldown antibodies, Kir4.1 was recovered (last panel). In all experiments when the pulldown antibody was omitted and lysates were incubated with protein A-Sepharose only (pA lanes) Kir4.1 was not recovered. Numbers on the right refer to the relative electrophoretic mobility of pre-stained molecular mass standards.

Connors and Kofuji, 2002), although incomplete denaturation usually occurs. As a result, we frequently see multiple bands corresponding to the four possible quarternary arrangements of channel subunits. The monomers tend to migrate around $43 \mathrm{kDa}$, which is close to the molecular weight of heavy-chain immunoglobulins as well as other nonspecific bands, so for clarity we show the tetrameric band, which runs at $\sim 180 \mathrm{kDa}$. When we performed immunoprecipitation for Kir4.1 then probed the blot for the channel, a band slightly under $200 \mathrm{kDa}$ was seen (Fig. 3, first panel) which is close to the calculated molecular weight for tetrameric Kir4.1 and is in agreement with prior studies (Li et al., 2001; Connors and Kofuji, 2002).

To verify whether we could recover Kir4.1 by pulling down DGC proteins, retinal lysates were first precipitated with anti- $\beta$-dystroglycan antibodies. When lysates from this experiment were probed with anti-Kir4.1, a band slightly under $200 \mathrm{kDa}$ (Fig. 3, second panel) was observed, indicating the recovery of the tetrameric form of the channel. We then used anti- $\alpha$-syntrophin antibodies for IP, which also resulted in the recovery of tetrameric Kir4.1 (Fig. 3, third panel). In both IPs, the omission of the pulldown antibody did not result in the recovery of Kir4.1.

The results outlined thus far suggest that Kir4.1 can co-precipitate with a number of DGC proteins in the retina. These proteins include Dp71 (and possibly Dp140), $\alpha$-syntrophin and perhaps other syntrophin isoforms, $\beta$ dystroglycan, and dystrobrevin. These results provide strong evidence that Kir4.1 forms a complex with DGC components in Müller cells.

\section{AQP4 Interacts With Kir4.1 and DGC in Retina}

A shared localization mechanism for Kir4.1 and AQP4 in retina is suggested by the tight co-localization of these 

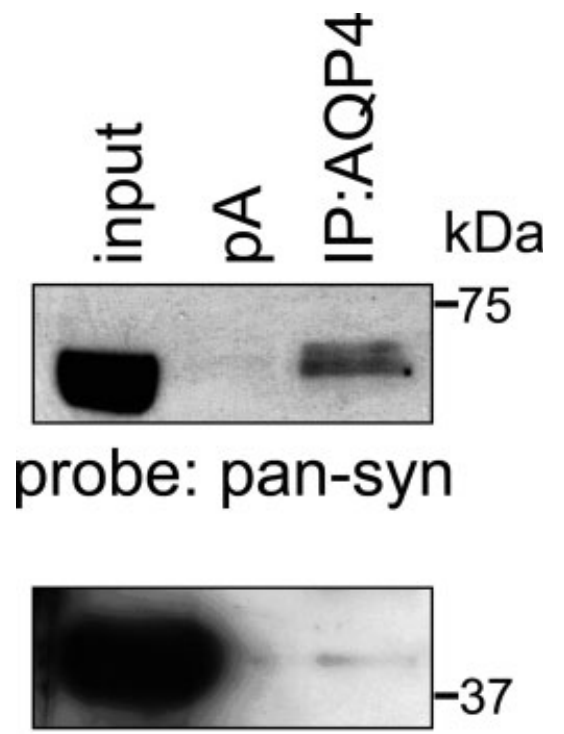

\section{probe: $\beta-D G$}

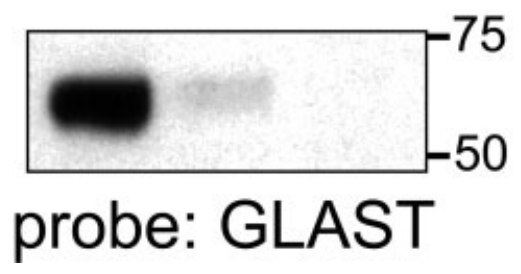

Fig. 4. AQP4 co-precipitates with DGC components in retina. Top: Anti-AQP4 antibodies were used to immunoprecipitate retinal lysates and the Western blot was probed with pan-syntrophin antibodies, revealing a band at $\sim 58 \mathrm{kDa}$ (last lane). This band was not when precipitated with protein A-Sepharose alone (middle lane). Middle: Same experiment was performed, but the blot was probed with anti $\beta$-dystroglycan antibodies, revealing a band in the IP lane (IP:AQP4) that is not seen in the protein A-Sepharose (pA), suggesting that AQP4 and $\beta$-dystroglycan form a complex in retina. Bottom: Antibodies on the blot shown in A were stripped and the blot was reprobed with anti-GLAST antibodies to determine whether the use of the crosslinking reagent, DSP, may introduce artifactual interactions. GLAST is seen in the input lane migrating at $\sim 63 \mathrm{kDa}$, but was not recovered by the IP reaction (IP:AQP4), suggesting that the interactions between AQP4 and the DGC are specific. Numbers on the right refer to the relative electrophoretic mobility of pre-stained molecular mass standards.

proteins in the endfeet and perivascular processes of Müller cells shown in Figure 1 and in prior studies (Nagelhus et al., 1999). Furthermore, AQP4 was shown to interact with the DGC in Müller cells (Dalloz et al., 2003) and brain astrocytes (Amiry-Moghaddam et al., 2003a, 2004; Neely et al., 2001). Based on this evidence, experiments were conducted to examine whether AQP4 can interact with DGC proteins and also with Kir4.1 in retina.

Based on the previously shown interactions between AQP4 and $\alpha$-syntrophin in brain (Amiry-Moghaddam et al., 2003a; Neely et al., 2001), AQP4 was first immunoprecipitated from rat retinal lysate, then probed with pan-syntrophin antibodies. The results of this experiment show that a protein slightly less than $75 \mathrm{kDa}$ was recovered (Fig. 4, top, IP:AQP4 lane). This finding suggests that AQP4 forms a complex with syntrophin in the retina. Anti-AQP4 immunoprecipitates were also probed for the

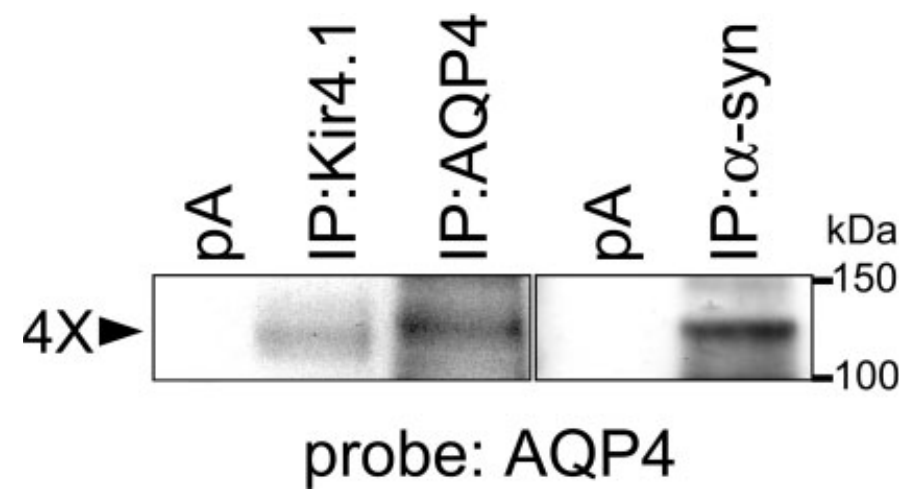

Fig. 5. AQP4 co-precipitates with $\alpha$-syntrophin and Kir4.1 in retina. Left: retinal tissue was immunoprecipitated with antibodies against Kir4.1 (IP:Kir4.1) and AQP4 (IP:AQP4) then probed with anti AQP4 antibodies. The first lane (pA) shows that the protein A-Sepharose matrix did not recover AQP4 alone. The second lane shows a band at $\sim 120 \mathrm{kDa}$ which corresponds to the tetrameric form of AQP4. The electrophoretic migration of AQP4 was verified to result in a band at $120 \mathrm{kDa}$ when AQP4 was immunoprecipitated and the blot was probed with anti-AQP4 antibodies. When anti- $\alpha$-syntrophin (right) were used as pulldown antibodies, a band at $\sim 120 \mathrm{kDa}$ was revealed after probing with antibodies against AQP4, suggesting that the water channel also associates with this protein in retina. Numbers on the right refer to the relative electrophoretic mobility of pre-stained molecular mass standards.

presence of the $43 \mathrm{kDa}$ transmembrane DGC component, $\beta$-dystroglycan. When antibodies against $\beta$-dystroglycan were used to probe the blot, a band slightly larger than $37 \mathrm{kDa}$ was seen in the IP lane (Fig. 4, middle), suggesting that AQP4 also interacts with $\beta$-dystroglycan in the retina.

To determine whether the interaction between AQP4 and syntrophin is mediated specifically by the $\alpha$ isoform of syntrophin in retina, anti- $\alpha$-syntrophin antibodies were used for IP of rat retina lysate. When the blots were probed with anti-AQP4, a band of 100-150 kDa was revealed (Fig. 5, right). Prior studies showed that under certain conditions, including with the use of the crosslinker DSP, AQP4 migrates as a tetramer (Neely et al., 1999). Figure 5 (left) shows that when AQP4 is immunoprecipitated and then probed with anti-AQP4 antibodies, a band is seen at $100-150 \mathrm{kDa}$, which is consistent with the expected molecular weight of the tetrameric form of this channel.

One concern about the use of chemical crosslinkers is the possible introduction of artifactual interactions. To address this issue, antibodies were chemically stripped from the blot shown in Figure 4 (top); the membrane was then reprobed with antibodies against GLAST. A GLAST immunopositive band can be seen at $50-75 \mathrm{kDa}$ in the lysate, however, is undetectable in the lane corresponding to the IP of AQP4 (Fig. 4, bottom, last lane). This demonstrates that these two membrane proteins were not induced to interact nonspecifically and suggests that DSP does not promiscuously introduce protein-protein interactions at the cell membrane.

Since the above data suggest that Kir4.1 and AQP4 can each interact with members of the DGC in retina, the next goal was to address whether Kir4.1 and AQP4 might interact through a common linkage with the DGC. To address this hypothesis, AQP4 was first immunoprecipitated from retinal lysate, which was then probed with 
anti-Kir4.1 antibodies. As shown in Figure 3 (last panel), the tetrameric band of Kir4.1 slightly less than $200 \mathrm{kDa}$ was revealed from the precipitated lysate, suggesting that these two channels can form a complex. The reverse experiment was also performed: the IP of Kir4.1 and probe for AQP4 resulted in the detection of a band of 100 $150 \mathrm{kDa}$, indicative of the tetrameric band of AQP4 (Fig. 5, left). Collectively, the evidence from the above experiments suggests that Kir4.1, AQP4, and the DGC can form a multi-protein complex in the retina.

\section{DISCUSSION}

Prior studies used cultured Müller cells to characterize the DGC in vitro (Claudepierre et al., 2000). Our data extends that knowledge by using tissue from intact retina to further characterize DGC interactions in vivo, and by examining the ability for the this complex to play a role in transmembrane channel localization. Using IP assays, we found compelling evidence for associations in the retina between Kir4.1 and a number of dystrophin-glycoprotein complex components, including dystrophin, $\alpha$-syntrophin, $\beta$-dystroglycan and dystrobrevin. We also provided evidence that the water channel AQP4 can co-precipitate with DGC proteins and Kir4.1. Together, these findings suggest a model whereby both channels are linked through the DGC, and further describe one of the few known mechanisms of subcellular localization of ion channels in glial cells.

The study of dystrophins is complicated by the large number of dystrophin isoforms expressed in vivo and their variable tissue distributions. In total, there are $\sim 18$ different dystrophins, which include splice variants of the major isoforms: Dp427, Dp260, Dp140, Dp116, and Dp71. All dystrophin isoforms have been identified within the CNS, and most can be found within the eye (Mehler, 2000). In the retina, Dp427 and Dp260 are localized to photoreceptor terminals in the OPL (D'Souza et al., 1995; Howard et al., 1998), while Dp140 and a particular isoform of Dp71 are likely expressed in retinal astrocytes (Claudepierre et al., 1999). It was reported that Müller cells also express a specific Dp71 isoform lacking exon 78, known as Dp71f (Claudepierre et al., 1999). However, one study showed that antibodies produced against a purported astrocyte-specific isoform can actually react with the Dp71 in Müller cells (Howard et al., 1998), suggesting that there may still be some ambiguity with regard to the distribution of specific Dp71 isoforms throughout the retina. Our findings that Kir4.1 can co-precipitate with Dp71 as well as Dp140 suggests that either Dp140 is expressed in Müller cells or that Kir4.1 is expressed elsewhere in the retina, such as in astrocytes of the nerve fiber layer, where Dp140 may be expressed (Claudepierre et al., 1999). Unfortunately due to the intensity of Kir4.1 immunostaining in Müller cell endfeet, it is impossible at the light microscopic level to discern astrocytic Kir4.1 staining from that found in Müller cell endfeet.

Along with the expression of multiple dystrophins, further DGC complexity arises from the number of syntro- phin isoforms that have been identified. Aside from $\alpha$-syntrophin, the ubiquitously expressed $\beta_{1}$ and $\beta_{2}$ (Ahn et al., 1996) and the brain-specific $\gamma_{1}$ and $\gamma_{2}$ isoforms (Piluso et al., 2000) have all been characterized. Furthermore, recent studies showed that there can be up to four distinct syntrophin binding sites within a single DGC, which can theoretically bind any combination of syntrophin (Peters et al., 1997; Newey et al., 2000). The differential expression patterns of these syntrophins within the CNS are still under investigation. It is tempting to speculate that the recruitment of particular molecules to the DGC could be regulated by the arrangement of syntrophin isoforms present. The number of dystrophins and syntrophins that are expressed make for the possibility of an elaborate matrix of interactions between the DGC, Kir4.1, and AQP4 within the CNS.

Over the last couple of years, research in this area has revealed an apparent interdependence between syntrophins, Kir4.1 and AQP4. For example, all three proteins tend to be upregulated in a parallel fashion in human astrocytes after a number of pathological brain states (Saadoun et al., 2003). These two channels plus $\alpha$-syntrophin and other DGC members also aggregate in glia when presented with laminin in cell culture (Guadagno and Moukhles, 2004). In addition, immunolocalization studies have shown that Kir4.1 and AQP4 are tightly co-localized in rat retina (Nagelhus et al., 1999), and AQP4 localization in astrocytes appears to be highly dependent on $\alpha$-syntroin (Inoue et al., 2002). Direct interactions between AQP4, $\alpha$-syntrophin, Dp71 and $\beta$-dystroglycan have been shown by IP assays (Neely et al., 2001; Amiry-Moghaddam et al., 2003a,b). Finally, the specific loss of Dp71 eliminates the polarized distribution of both Kir4.1 and AQP4 in retina (Dalloz et al., 2003). Overall, these reports describe a convincing body of evidence that Kir4.1, AQP4 and DGC proteins are closely interrelated in some way.

Despite the apparent relationship between these proteins, there may be differences in the way Kir4.1 and AQP4 interact with the DGC. For example, immunoelectron microscopy showed that the absence of $\alpha$-syntrophin dramatically altered the localization pattern of AQP4 but not Kir4.1 in hippocampal astrocytes (Amiry-Moghaddam et al., 2003a). Similar evidence has been proposed in retinas from $\alpha$-syntrophin null mice where the localization of AQP4 was marginally decreased in Müller cell endfeet and perivascular processes, whereas Kir4.1 localization was unaffected (Frydenlund et al., 2004).

Biochemical studies have demonstrated that there may be specificity of interactions between particular syntrophins and their binding partners. This idea is lent credence by the findings that PDZ domains can discriminate interaction partners based on single amino acid substitutions (Songyang et al., 1997; Gee et al., 2000; Vaccaro et al., 2001). Therefore, the differences in the PDZ domain-binding C-termini of Kir4.1 (-R-I-S-N-V) and AQP4 (-V-L-S-S-V) may facilitate the preferential binding of AQP4 to a particular syntrophin isoform and Kir4.1 to another. Moreover, slight sequence differences within the PDZ domains of syntrophins may also purvey selectivity for particular binding partners. 
The interactions between the DGC, Kir4.1, and AQP4 may influence the efficiency of the glial $\left[\mathrm{K}^{+}\right]_{\mathrm{o}}$ buffering function. Modeling studies (Odette and Newman, 1988) suggest that the enrichment of $\mathrm{K}^{+}$channels at the endfeet of Müller cells enhance $\mathrm{K}^{+}$buffering. Our studies show a redistribution of $\mathrm{K}^{+}$conductances in the $\mathrm{mdx}^{3 C v}$ mouse which could potentially alter $\mathrm{K}^{+}$fluxes in retina. There is also evidence that AQP4 is involved in $\left[\mathrm{K}^{+}\right]_{\mathrm{o}}$ buffering. First, the volume of the extracellular space in brain appears to be negatively correlated with $\left[\mathrm{K}^{+}\right]_{0}$, suggesting the involvement of a water transport mechanism (Dietzel et al., 1980; Holthoff and Witte, 2000). Second, $\alpha$-syntrophin null mice show an impairment of both water transport and $\mathrm{K}^{+}$buffering in brain resulting from a redistribution of AQP4 away from astrocytic endfeet (AmiryMoghaddam et al., 2003b, 2004). Finally, following an experimentally induced loss of Kir4.1 in retina, ischemic conditions resulted in increased cell swelling under hypoosmotic conditions (Pannicke et al., 2004). As a group, these data suggest that water and $\mathrm{K}^{+}$transport are tightly coupled, which could stem from the co-associations of Kir4.1 and AQP4 with the DGC.

\section{ACKNOWLEDGMENTS}

The authors are very grateful to Sara Tauer and Terry Wu for excellent technical assistance. This work was supported by National Institutes of Health grant EY12949 (to P.K.) and by a Vision Training Grant EY07133 (to N.C.C.).

\section{REFERENCES}

Adams ME, Dwyer TM, Dowler LL, White RA, Froehner SC. 1995. Mouse alpha 1- and beta 2-syntrophin gene structure, chromosome localization, and homology with a discs large domain. J Biol Chem 270:25859-25865.

Ahn AH, Freener CA, Gussoni E, Yoshida M, Ozawa E, Kunkel LM. 1996. The three human syntrophin genes are expressed in diverse tissues, have distinct chromosomal locations, and each bind to dystrophin and its relatives. J Biol Chem 271:2724-2730.

Amedee T, Robert A, Coles JA. 1997. Potassium homeostasis and glial energy metabolism. Glia 21:46-55.

Amiry-Moghaddam M, Otsuka T, Hurn PD, Traystman RJ, Haug FM, Froehner SC, Adams ME, Neely JD, Agre P, Ottersen OP, Bhardwaj A. 2003a. An alpha-syntrophin-dependent pool of AQP4 in astroglial end-feet confers bidirectional water flow between blood and brain. Proc Natl Acad Sci USA 100:2106-2111.

Amiry-Moghaddam M, Williamson A, Palomba M, Eid T, de Lanerolle NC, Nagelhus EA, Adams ME, Froehner SC, Agre P, Ottersen OP. 2003 b. Delayed $\mathrm{K}^{+}$clearance associated with aquaporin-4 mislocalization: phenotypic defects in brains of alpha-syntrophin-null mice. Proc Natl Acad Sci USA 100:13615-13620.

Amiry-Moghaddam M, Xue R, Haug FM, Neely JD, Bhardwaj A, Agre P, Adams ME, Froehner SC, Mori S, Ottersen OP. 2004. Alpha-syntrophin deletion removes the perivascular but not endothelial pool of aquaporin-4 at the blood-brain barrier and delays the development of brain edema in an experimental model of acute hyponatremia. FASEB J 18:542-544.

Austin RC, Howard PL, D'Souza VN, Klamut HJ, Ray PN. 1995. Cloning and characterization of alternatively spliced isoforms of Dp71. Hum Mol Genet 4:1475-1483.

Claudepierre T, Dalloz C, Mornet D, Matsumura K, Sahel J, Rendon A. 2000. Characterization of the intermolecular associations of the dystrophin-associated glycoprotein complex in retinal Müller glial cells. J Cell Sci 113:3409-3417.

Claudepierre T, Rodius F, Frasson M, Fontaine V, Picaud S, Dreyfus H, Mornet D, Rendon A. 1999. Differential distribution of dystrophins in rat retina. Invest Ophthalmol Vis Sci 40:1520-1529.
Connors NC, Kofuji P. 2002. Dystrophin Dp71 is critical for the clustered localization of potassium channels in retinal glial cells. J Neurosci 22:4321-4327.

Connors NC, Adams ME, Froehner SC, Kofuji P. 2004. The potassium channel Kir4.1 associates with the dystrophin-glycoprotein complex via alpha-syntrophin in glia. J Biol Chem 279:28387-28392.

Dalloz C, Sarig R, Fort P, Yaffe D, Bordais A, Pannicke T, Grosche J, Mornet D, Reichenbach A, Sahel J, Nudel U, Rendon A. 2003. Targeted inactivation of dystrophin gene product Dp71: phenotypic impact in mouse retina. Hum Mol Genet 12:1543-1554.

Dietzel I, Heinemann U, Hofmeier G, Lux HD. 1980. Transient changes in the size of the extracellular space in the sensorimotor cortex of cats in relation to stimulus-induced changes in potassium concentration. Exp Brain Res 40:432-439.

D'Souza VN, Nguyen TM, Morris GE, Karges W, Pillers DA, Ray PN. 1995. A novel dystrophin isoform is required for normal retinal electrophysiology. Hum Mol Genet 4:837-842.

Froehner SC, Murnane AA, Tobler M, Peng HB, Sealock R. 1987. A postsynaptic $\mathrm{Mr} 58,000(58 \mathrm{~K})$ protein concentrated at acetylcholine receptor-rich sites in Torpedo electroplaques and skeletal muscle. J Cell Biol 104:1633-1646.

Frydenlund DS, Puwarawuttipanit W, Bragg A, Adams ME, Haug FM, Kotchabhakdi N, Ottersen OP. 2004. Effects of alpha-syntrophin knockout on expression of AQP4 and Kir4.1 in retina. San Diego, CA: Society for Neuroscience Meeting. Abstract 405.22.

Gee SH, Quenneville S, Lombardo CR, Chabot J. 2000. Single-amino acid substitutions alter the specificity and affinity of PDZ domains for their ligands. Biochemistry 39:14638-14646.

Guadagno E, Moukhles H. 2004. Laminin-induced aggregation of the inwardly rectifying potassium channel, Kir4.1, and the water-permeable channel, AQP4, via a dystroglycan-containing complex in astrocytes. Glia 47:138-149.

Holthoff K, Witte OW. 2000. Directed spatial potassium redistribution in rat neocortex. Glia 29:288-292.

Howard PL, Dally GY, Wong MH, Ho A, Weleber RG, Pillers DA, Ray PN. 1998. Localization of dystrophin isoform Dp71 to the inner limiting membrane of the retina suggests a unique functional contribution of Dp71 in the retina. Hum Mol Genet 7:1385-1391.

Inoue M, Wakayama Y, Liu JW, Murahashi M, Shibuya S, Oniki H. 2002. Ultrastructural localization of aquaporin 4 and alpha1-syntrophin in the vascular feet of brain astrocytes. Tohoku J Exp Med 197:87-93.

Kofuji P, Connors NC. 2003. Molecular substrates of potassium spatial buffering in glial cells. Mol Neurobiol 28:195-208.

Kofuji P, Ceelen P, Zahs KR, Surbeck LW, Lester HA, Newman EA. 2000. Genetic inactivation of an inwardly rectifying potassium channel (Kir4.1 subunit) in mice: phenotypic impact in retina. J Neurosci 20:5733-5740.

Li L, Head V, Timpe LC. 2001. Identification of an inward rectifier potassium channel gene expressed in mouse cortical astrocytes. Glia 33:57-71.

Mehler MF. 2000. Brain dystrophin, neurogenetics and mental retardation. Brain Res Brain Res Rev 32:277-307.

Michele DE, Campbell KP. 2003. Dystrophin-glycoprotein complex: post-translational processing and dystroglycan function. J Biol Chem 278:15457-15460.

Moore SA, Saito F, Chen J, Michele DE, Henry MD, Messing A, Cohn RD, Ross-Barta SE, Westra S, Williamson RA, Hoshi T, Campbell KP. 2002. Deletion of brain dystroglycan recapitulates aspects of congenital muscular dystrophy. Nature 418:422-425.

Nagelhus EA, Horio Y, Inanobe A, Fujita A, Haug FM, Nielsen S, Kurachi Y, Ottersen OP. 1999. Immunogold evidence suggests that coupling of $\mathrm{K}^{+}$siphoning and water transport in rat retinal Müller cells is mediated by a coenrichment of Kir4.1 and AQP4 in specific membrane domains. Glia 26:47-54.

Nagelhus EA, Mathiisen TM, Ottersen OP. 2004. Aquaporin-4 in the central nervous system: cellular and subcellular distribution and coexpression with Kir4.1. Neuroscience 129:905-913.

Neely JD, Christensen BM, Nielsen S, Agre P. 1999. Heterotetrameric composition of aquaporin-4 water channels. Biochemistry 38:11156-11163.

Neely JD, Amiry-Moghaddam M, Ottersen OP, Froehner SC, Agre P, Adams ME. 2001. Syntrophin-dependent expression and localization of Aquaporin-4 water channel protein. Proc Natl Acad Sci USA 98:14108-14113

Newey SE, Benson MA, Ponting CP, Davies KE, Blake DJ. 2000. Alternative splicing of dystrobrevin regulates the stoichiometry of syntrophin binding to the dystrophin protein complex. Curr Biol 10:1295-1298.

Newman E, Reichenbach A. 1996. The Müller cell: a functional element of the retina. Trends Neurosci 19:307-312.

Newman EA, Odette LL. 1984. Model of electroretinogram b-wave generation: a test of the $\mathrm{K}^{+}$hypothesis. J Neurophysiol 51:164-182.

Odette LL, Newman EA. 1988. Model of potassium dynamics in the central nervous system. Glia 1:198-210. 
Pannicke T, Iandiev I, Uckermann O, Biedermann B, Kutzera F, Wiedemann P, Wolburg H, Reichenbach A, Bringmann A. 2004. A potassium channel-linked mechanism of glial cell swelling in the postischemic retina. Mol Cell Neurosci 26:493-502.

Peters MF, O'Brien KF, Sadoulet-Puccio HM, Kunkel LM, Adams ME, Froehner SC. 1997. beta-dystrobrevin, a new member of the dystrophin family. Identification, cloning, and protein associations. J Biol Chem 272:31561-31569.

Pillers DA, Weleber RG, Green DG, Rash SM, Dally GY, Howard PL, Powers MR, Hood DC, Chapman VM, Ray PN, Woodward WR. 1999a. Effects of dystrophin isoforms on signal transduction through neural retina: genotype-phenotype analysis of Duchenne muscular dystrophy mouse mutants. Mol Genet Metab 66:100-110.

Pillers DM, Fitzgerald KM, Duncan NM, Rash SM, White RA, Dwinnell SJ, Powell BR, Schnur RE, Ray PN, Cibis GW, Weleber RG. 1999b. Duchenne/Becker muscular dystrophy: correlation of phenotype by electroretinography with sites of dystrophin mutations. Hum Genet 105:2-9.

Piluso G, Mirabella M, Ricci E, Belsito A, Abbondanza C, Servidei S, Puca AA, Tonali P, Puca GA, Nigro V. 2000. Gamma1- and gamma2syntrophins, two novel dystrophin-binding proteins localized in neuronal cells. J Biol Chem 275:15851-15860.
Saadoun S, Papadopoulos MC, Krishna S. 2003. Water transport becomes uncoupled from $\mathrm{K}^{+}$siphoning in brain contusion, bacterial meningitis, and brain tumours: immunohistochemical case review. J Clin Pathol 56:972-975.

Sadoulet-Puccio HM, Rajala M, Kunkel LM. 1997. Dystrobrevin and dystrophin: an interaction through coiled-coil motifs. Proc Natl Acad Sci USA 94:12413-12418.

Songyang Z, Fanning AS, Fu C, Xu J, Marfatia SM, Chishti AH, Crompton A, Chan AC, Anderson JM, Cantley LC. 1997. Recognition of unique carboxyl-terminal motifs by distinct PDZ domains. Science 275:73-77.

Suzuki A, Yoshida M, Ozawa E. 1995. Mammalian alpha 1- and beta 1syntrophin bind to the alternative splice-prone region of the dystrophin $\mathrm{COOH}$ terminus. J Cell Biol 128:373-381.

Vaccaro P, Brannetti B, Montecchi-Palazzi L, Philipp S, Citterich MH, Cesareni G, Dente L. 2001. Distinct binding specificity of the multiple PDZ domains of INADL, a human protein with homology to INAD from Drosophila melanogaster. J Biol Chem 276:42122-42130.

Zhu T, Dahan D, Evagelidis A, Zheng S, Luo J, Hanrahan JW. 1999. Association of cystic fibrosis transmembrane conductance regulator and protein phosphatase 2C. J Biol Chem 274:29102-29107. 